УДК 621.314

\title{
ГАРМОНИЧЕСКИЙ СОСТАВ ВЫХОДНОГО НАПРЯЖЕНИЯ ВЫПРЯМИТЕЛЯ С ШИРОТНО - ИМПУЛЬСНОЙ МОДУЛЯЦИЕЙ
}

Канд. техн. наук Е.Я. Ивакина

\section{ГАРМОНІЙНИЙ СКЛАД ВИХІДНОЇ НАПРУГИ ВИПРЯМЛЯЧА 3 ШИРОТНО - ІМПУЛЬСНОї МОДУЛЯЦІї}

Канд. техн. наук К.Я. Івакіна

\section{THE HARMONIC COMPOSITION OF THE OUTPUT VOLTAGE OF THE RECTIFIER WITH LATITUDINAL-IMPULSIVE MODULATION}

\author{
Cand. of techn. sciences K.Y. Ivakina
}

В статье выполнен анализ гармонического состава выходного напряжения выпрямителя с широтно-импульсной модуляцичей. Получено выражение для комплексной величины q-й гармоники. Приведено графическое отображение

$q-\tilde{u}$ гармоники выходного напряжения вольтодобавочного преобразователя на комплексной плоскости, что позволяет наглядно иллюстрировать процесс их образования.

Ключевые слова. Выпрямитель, широтно-импульсная модуляциия, гармонический состав, вольтодобавочный преобразователь, векторная диаграмма.

У статті виконано аналіз гармонійного складу вихідної напруги випрямляча з широтно-імпульсною модуляиією. Отримано вираз для комплексної величини q -й гармоніки. Наведено графічне відображення q-й гармоніки вихідної напруги вольтододатного перетворювача на комплексній площині, щэо дозволяє наочно ілюструвати процес їх утворення.

Ключові слова. Випрямляч, широтно-імпульсна модулячія, гармонійний склад, вольтододатний перетворювач, векторна діаграма.

In the article the analysis of harmonic composition of the output voltage of the rectifier with latitudinal-impulsive modulation, is executed. The analysis of harmonic composition is executed for the system of automatic control of output tension of rectifier with an integral regulator. The expression for the complex quantity $q$ - th harmonic obtained. The graphic image of q-th harmonic of the output voltage of the rectifier with latitudinal-impulsive modulation in the complex plane submitted. This graphic image illustrates the process of their education.

Keywords. Rectifier, latitudinal-impulsive modulation, harmonic composition, booster converter, vector diagram. 
1. Вступление. Улучшение качества электрической энергии на выходе мощной системы питания, которая построена на базе выпрямителя, связано с оптимизацией гармонического состава выходного напряжения. Известна эффективность применения для этой цели замкнутых систем автоматического регулирования.

2. Постановка проблемы в общем виде и ее связь с важными научными и практическими заданиями. Повышение качества электрической энергии на выходе полупроводниковых преобразователей путем применения замкнутых систем автоматического регулирования является актуальной задачей. Данная проблема непосредственно связана с улучшением электромагнитной совместимости тяговых подстанций постоянного тока с контактной сетью. Применение в выпрямителе широтно-импульсной модуляции для регулирования среднего значения выходного напряжения одновременно с расширением полосы пропускания изменяет природу генерации гармоник по сравнению с фазовым регулированием. В связи с этим возникает необходимость исследования процесса генерации гармонических составляющих в выходное напряжение выпрямителя с широтно-импульсной модуляцией.

3. Анализ последних достижений и публикаций. В работах $[1,2]$ приведены результаты исследований выпрямителей с широтно - импульсной модуляцией. Однако данные исследования имеют ограниченный характер, заключающийся в рассмотрении их основных энергетических показателей.

4. Цель и задачи исследований. Задачей исследований является определения особенностей влияния широтно импульсной модуляции на формирования гармонического состава выходного напряжения выпрямителя. Целью исследований - получение аналитических

$$
U_{u}\left(\theta_{n T_{2}}\right)=U_{m} \sin \left[\frac{2 \pi}{m}-(n-1) \frac{2 \pi}{K_{o} m}+\gamma \frac{\pi}{K_{o} m}\right]=U_{m} \sin \left\{\frac{\pi}{K_{o} m}\left[2\left(1-n+K_{o}\right)+\gamma\right]\right\}
$$

зависимостей, гармонический определяющих напряжения выпрямителя с широтно импульсной модуляцией при симметрии питающих напряжений.

\section{5. Основная часть исследований.} Полоса пропускания шести и двенадцатипульсного тиристорных выпрямителей, которые широко применяют для построения мощных стабилизированных систем питания, во многих случаях является недостаточной.

Использование B выпрямителе полностью управляемых силовых ключей позволяет вместо фазового управления применить широтно- импульсное регулирование выходного напряжения $[1,3$, 4]. Применение современных силовых ключей позволяет реализовать в выпрямителе с достаточно малыми потерями широтно-импульсную модуляцию (ШИМ) в несколько килогерц [5]. Это позволяет значительно расширить его полосу пропускания.

При

рассмотрении гармонического состава выходного напряжения выпрямителя с ШИМ приняты допущения о симметрии питающей сети, идеальности системы управления и силовых ключей.

Выходное напряжение выпрямителя с ШИМ (см. рис. 1) представляет собой последовательность импульсов длительностью $\theta_{\gamma}$, ограниченных сверху линейными напряжениями выпрямителя. В [6] показано, что данная импульсная последовательность может быть, с достаточно высокой степенью точности, аппроксимирована последовательностью прямоугольных импульсов с амплитудами 


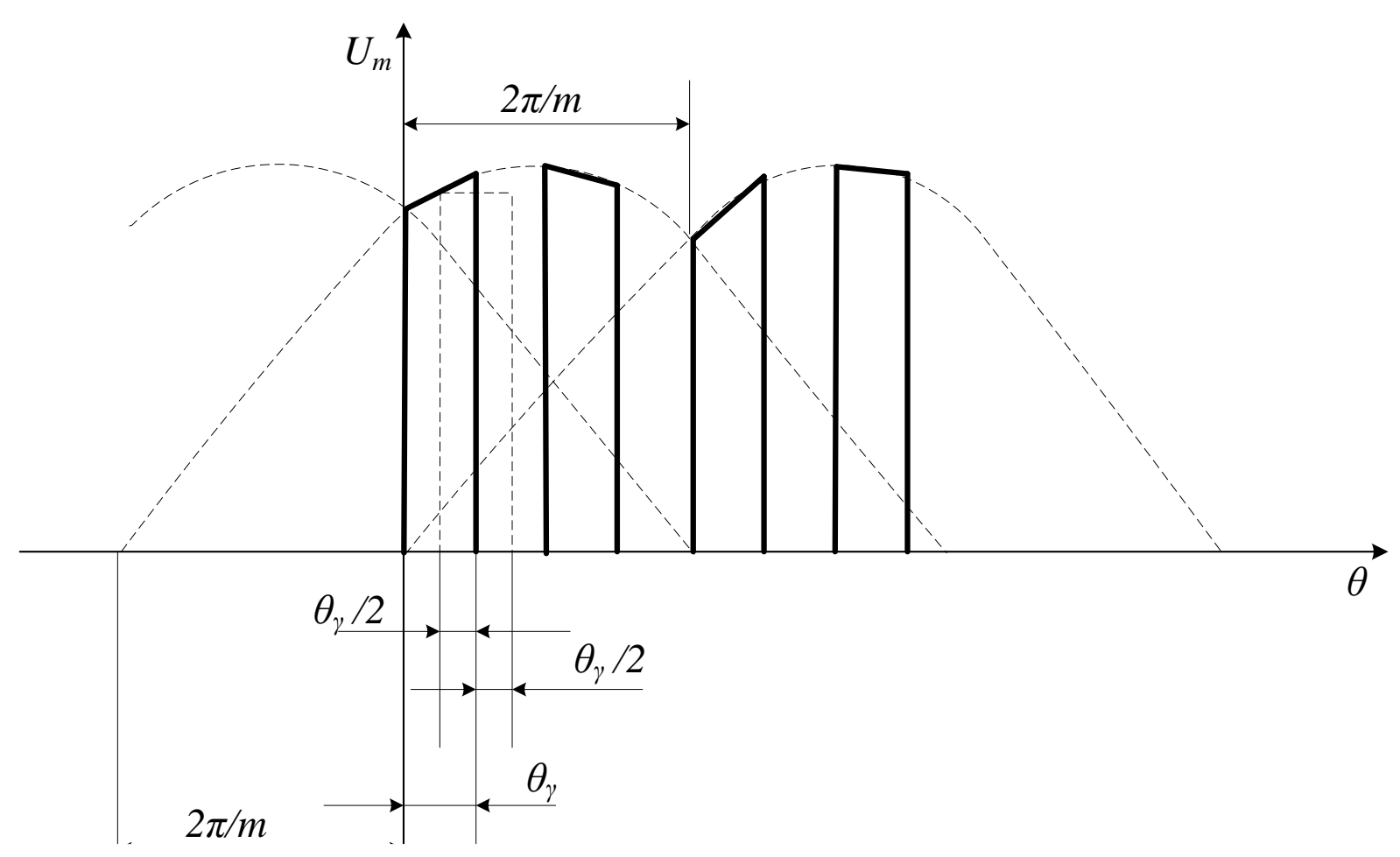

Рис. 1- Выходное напряжения выпрямителя с ШИМ

Комплексную величину $q$-й гармоники определим разложением в ряд Фурье импульсной последовательности (1)

$$
\dot{U}_{q}=\frac{m}{\pi} \sum_{n=1}^{K_{O}} \int_{-\gamma \frac{\pi}{K_{O} m}+\varphi_{H}+\frac{2 \pi}{K_{O} m}(n-1)}^{\gamma \frac{\pi}{K_{O} m}+\varphi_{H}+\frac{2 \pi}{K_{O} m}(n-1)} U_{m} \sin \left\{\frac{\pi}{K_{o} m}\left[2\left(1-n+K_{o}\right)+\gamma\right]\right\} e^{-j q K_{O} \theta} d \theta
$$

Для получения однозначности фазовой привязки гармоник, в выражении (2) угловая координата $\varphi_{н}$ зависит от вида соединений обмоток трансформаторов, питающих выпрямитель с ШИМ. При соединениях звезда - звезда угол $\varphi=0$. При соединениях звезда треугольник угол $\varphi=\frac{\pi}{6}$.

Вычислив в (2) интеграл и подставив пределы интегрирования, получим

$$
\begin{gathered}
\dot{U}_{q}=-\frac{U_{m} m}{j \pi q K_{o}} \sum_{n=1}^{K_{o}}\left[e^{-j q K_{O}\left(\frac{2 \pi}{K_{o} m}(n-1)+\gamma \frac{\pi}{K_{o} m}-\varphi_{H}\right)}-e^{-j q K_{O}\left(\frac{2 \pi}{K_{o} m}(n-1)-\gamma \frac{\pi}{K_{o} m}-\varphi_{H}\right)}\right] \times \\
\quad \times \sin \left\{\frac{\pi}{K_{o} m}\left[2\left(n-1+K_{o}\right)+\gamma\right]\right\}
\end{gathered}
$$

Преобразуем в (3)

$$
e^{-j q \frac{\pi}{m}}-e^{j q \frac{\pi}{m}}=\left[-j \sin q \gamma \frac{\pi}{m}-j \sin q \gamma \frac{\pi}{m}\right]=-2 j \sin q \gamma \frac{\pi}{m}
$$


С учетом (4) выражение (3) для комплексной величины $q$ - й гармоники преобразуется к виду

$$
\dot{U}_{q}=\frac{2 U_{m} m}{\pi q K_{o}} \sin q \gamma \frac{\pi}{m} \sum_{n=1}^{K_{o}} \sin \frac{\pi}{K_{o} m}\left[2\left(1-n+K_{o}\right)+\gamma\right] e^{-j q K_{o}\left[\frac{2 \pi}{K_{o} m}(n-1)-\varphi_{H}\right]} .
$$

Перейдя к относительным единицам, приняв в качестве базовой величины среднее значение выпрямленного напряжения неуправляемого выпрямителя

$$
\begin{aligned}
& \mathrm{U}_{\mathrm{do}}=U_{m} \frac{m}{\pi} \sin \frac{\pi}{m}, \text { получим } \\
& \left.\dot{U}_{q}=\frac{2 \sin q \gamma \frac{\pi}{m} K_{o}}{q K_{o} \sin \frac{\pi}{m}} \sum_{n=1}^{m} \sin \frac{\pi}{K_{o} m}\left[2\left(1-n+K_{o}\right)+\gamma\right]\right\} e^{-j q K_{o}\left[\frac{2 \pi}{K_{o} m}(n-1)-\varphi_{H}\right]},
\end{aligned}
$$

где $q=1,2,3 \ldots$. номер гармоники.

Частота $q$ - й гармоники равна

$$
f_{q}=m \cdot q \cdot K_{o} \cdot f_{o} .
$$

Выражение (6) имеет наглядную графическую интерпретацию. Для этого построим на комплексной плоскости $K_{o}$ лучевую звезду, у которой центральные углы между соседними лучами

$$
\varphi_{n}=-q\left[\frac{2 \pi}{m}(1-n)-K_{o} \varphi_{H}\right]
$$

В тех случаях, когда $q$ и $m$ имеют общие делители, некоторые лучи накладываются друг на друга. Если теперь на каждом луче изобразить вектор длинной

$$
a_{n}=\frac{2 \sin q \gamma \frac{\pi}{m}}{q K_{o} \sin \frac{\pi}{m}} \sin \frac{\pi}{K_{o} m}\left[2\left(1-n+K_{o}\right)+\gamma\right]
$$

то геометрическая сумма всех $K_{o}$ оценить зависимость амплитуд гармоник в векторов и будет вектором $q$-й гармоники. функции относительной длительности Данное построение позволяет наглядно импульсов широтно-импульсной модуляции.

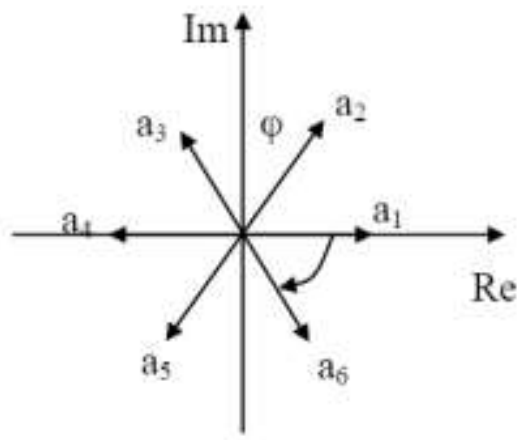

Рнс.2- Векторная диаграмма вектора $q$-й гармоники выходного напряжения 
Анализ выражения (8) показывает, гармоники выходного напряжения. что для трех фазной мостовой схемы Приведенная векторная диаграмма выпрямителя центральные углы между построена для следующих параметров: $m=$ соседними лучами кратны $\pi / 3$. На рис. $26, K_{o}=6, \varphi_{H}=0$. В этом случае центральные приведена обобщенная векторная диаграмма углы между соседними лучами составляющих результирующего вектора $q$-й определяются следующим образом

$$
\varphi_{n}=-q \frac{\pi}{3}(1-n)
$$

6. Выводи из исследований и информацию о гармониках вызываемых перспективы, дальнейшее развитие в пульсационной составляющей выпрямителя данном направлении. Установлено, что и широтно-импульсной модуляцией. гармонический состав выходного Показано, что отображение гармоник на напряжения выпрямителя с широтно- комплексной плоскости позволяет наглядно импульсной модуляцией содержит иллюстрировать процесс их образования.

\section{Список литературы}

1. Шавелкин А.А. Преобразовательная техника / А.А. Шавелкин, А.М.Наливайко// ДДМА.- Краматорск, 2008.-328c.

2. Обратимый преобразователь вольтодобавочного типа для тяговых подстанций электрифицированных железных дорог / Н.В. Панасенко, В.В. Божко, Ю.П. Гончаров [и др.] // Залізничний транспорт України. - 2007. - №4. - С.76-80.

3. Розанов Ю.К. Основы силовой электроники / Ю.К. Розанов. - М.: Энергоатомиздат, 1992.-296с.

4. Grzegorz Benysek, Marian Pasko Editors Power Theories for Improved Power Quality.- Springer-Verlag London 2012.-216p.

5. Wei L. Novel Matrix Converter Topology with Simple Commutation/ L.Wei, T. A Lipo // Record of the IEEE Industry Applications Sosiety Annual Meeting.- Chicago (USA).Sept.30-Oct.4,2001.-Vol.3.

6. Івакіна К.Я. Аналіз електромагнітних процесів випрямляча 3 широтноімпульсною модуляцією /К.Я.Івакіна// Вісник Вінницького політехнічного інституту. Вінниця, 2013. -№ 6. - С. 65-67.

Івакіна Катерина Яківна, канд. техн. наук, кафедра електричного транспорту Харківського національного університету міського господарства ім. O.M. Бекетова. E-mail: ivakina katya@mail.ru.

Ivakina Katerina Yakivna, candidate tehnichny Sciences. Department electric of transport Kharkiv National University of Municipal Management named after A.N. Beketova. E-mail: ivakina_katya@mail.ru.

Стаття поступила 20.05.2015 